\title{
CORRELATION OF MACROSCOPIC AND HISTOPATHOLOGIC FINDINGS WITH MRI IN FORENSIC EXAMINATIONS
}

\author{
Webb B ${ }^{1}$, Petrovic $A^{1}$ and Scheurer $E^{1}$ \\ ${ }^{1}$ Ludwig Boltzmann Institute for Clinical-Forensic Imaging, Austria \\ bridgette.webb@cfi.lbg.ac.at
}

\begin{abstract}
The present study investigated the visibility of a strand-shaped fiducial marker in MRI, macroscopic photography and histopathology with the aim of correlating images across these modalities. Eight small samples of porcine tissue were internally marked prior to undergoing MRI. Samples were then sectioned and processed in routine histopathology. Visual comparison of results was followed by measurement of the relative distances between markers. This enabled the correlation of specific MR images with histology slices. This study provides guidance for ongoing research into the forensic applications of the examined fiducial marker, both in post-mortem cases and in living victims.
\end{abstract}

Keywords: MRI, marker, histopathology, correlation, forensic.

\section{Introduction}

Objective and accurate forensic documentation of soft tissue injuries is essential in both clinical and postmortem forensic examinations. Subcutaneous soft tissue contains a lack of anatomical landmarks which, in other regions of the body, would otherwise be used to combine and evaluate information from various imaging modalities. Given this absence, and with a view of future forensic applications, this study investigated the potential of a strand-shaped fiducial marker to correlate soft tissue findings in MRI, macroscopic and histopathology examinations. Such a marker was previously used to register digital histology images to MR images in the prostate [1].

MRI has shown a particular utility in detecting and assessing traumatic lesions in subcutaneous fatty tissue including haematomas [2]. In post-mortem cases, forensic histopathology contributes to the histomorphological determination of injury age and the extent of tissue damage due to blunt force [3].

This study examined the ex vivo effectiveness of the selected fiducial marker in porcine tissue. Of specific interest, was the feasibility of the method, including visibility of markers across each of the techniques and the potential to determine the MR image corresponding to a given histopathology slide.

\section{Methods}

In the present study, strand-shaped fiducial markers were inserted into small samples $\left(2 \times 3 \times 1.6 \mathrm{~cm}^{3}\right)$ of porcine (ventral tissue). The samples were scanned by MRI and digitally registered by photography (tissue sections (TS images) and histopathology preparations (HP images)). Images from the different modalities were compared with one another.

\section{Sample Preparation}

Two sets of strand-shaped fiducial markers were prepared by soaking thread ( $100 \%$ cotton) in a $40: 1$ solution containing black tissue dye (Mark-It ${ }^{\mathrm{TM}}$, Richard-Allan Scientific, USA) and an MRI contrast agent (Gadovist (1.0 $\mathrm{mmol} / \mathrm{mL})$ or Magnevist gadolinium $(0.5 \mathrm{mmol} / \mathrm{mL}))$.

Eight samples (skin-fat-muscle-fat layers) were prepared. To simulate a finding in the sample, a small cavity was created from underneath, and a piece of muscle tissue inserted. Four to six threads were inserted from the front to the back of each sample in varying configurations (structured or random). The needle/cannula (20-G) was first inserted. Then, following removal of the needle, a pre-soaked thread was inserted through the cannula. Finally, the cannula was removed, leaving the marker in place.

\section{MR Imaging}

A Magnetom Skyra (Siemens AG, Germany) at 3T and a CPC 8-Channel coil (Noras GmbH, Germany) placed close to the sample were used to acquire images perpendicular to the tissue layers.

Table 1: Scan parameters* for acquiring MR images

\begin{tabular}{cccccc}
\hline Sequence & $\begin{array}{c}\text { TE } \\
(\mathbf{m s})\end{array}$ & $\begin{array}{c}\text { TR } \\
(\mathbf{m s})\end{array}$ & $\begin{array}{c}\text { FA } \\
(\mathbf{d e g})\end{array}$ & $\begin{array}{c}\text { Slice thickness } \\
(\mathbf{m m})\end{array}$ & $\begin{array}{c}\text { In-plane } \\
\text { Resolution } \\
(\mathbf{m m})\end{array}$ \\
\hline 2D TSE_T1w & 11 & 600 & - & 1.4 & 0.31 \\
2D TSE_T2w & 78 & 3000 & - & 1.4 & 0.31 \\
2D TSE_PDw & 11 & 3000 & - & 1.4 & 0.31 \\
Iso-3D GRE & 3.84 & 40 & 27 & 0.31 & 0.31 \\
Iso-3D GRE & 3.84 & 40 & 27 & 0.42 & 0.42 \\
\hline
\end{tabular}

*Field of view: $80 \mathrm{~mm}(100 \%)$

\section{Tissue Section [TS] Processing}

After scanning, samples were fixed in formalin (4\%). Threads were removed and each sample was sliced into small TS of $5 \mathrm{~mm}$ thickness. A single slice $(2-4 \mu \mathrm{m})$ from the face of each TS was then processed via routine histological staining (haematoxylin-eosin). The faces of the paraffin embedded TS and the corresponding $H \& E$ stained microscopic slides were photographed (macro lens; Lumix GF1). These images were treated to maximise visualisation of the sample edges and markers (Photoshop CS6, Adobe).

\section{Data Analysis}

Following visual comparison of the images (Fig. 1), the relative distance between markers (Tab. 2) was used to determine more precisely the correspondence of a MR image and physical sample slice. 


\section{Results}

Due to the varying rigidity of tissue layers within the sample (e.g. muscle-fat-skin), distortion during histological processing rendered some samples unsuitable for comparison.

In the TS, markers were clearly visible (Fig. 1 (a)) and in histology, markers placed entirely within a given layer (i.e. not at an interface) were visible due to the diffusion of dye into surrounding tissue (Fig. 1 (b)). The positional difference between TS and HP images was negligible $(\max .5 \mu \mathrm{m})$ in relation to MR slice thickness $(310 \mu \mathrm{m})$.

Using proton density weighting, good contrast both between tissue types (fatty tissue/muscle: grey/bright), and with the black markers was obtained. However, MR images acquired using $2 \mathrm{D}$ sequences were too thick to allow a precise correlation with histology slices as these slices were often located in a position between two MR images. The isotropic three-dimensional gradient-echo (GRE) sequence acquired sufficiently thin contiguous slices for a sample in less than 10 minutes (Fig. 1(c)-(e)). The choice of contrast agent did not influence the visibility of markers and no marker-related artefacts were observed. (a)

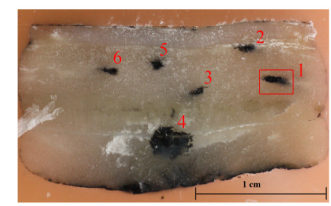

(b)

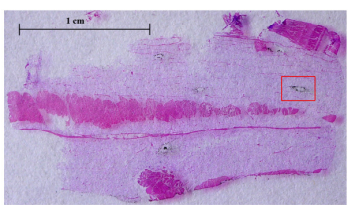

(c)

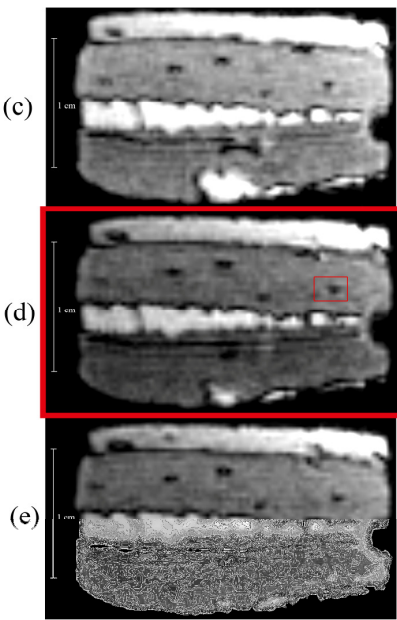

Figure 1: Comparison of images across modalities (a) TS image, markers 1-6 (b) HP image (c) - (e) MR images 1-3 (Tab. 2) (Iso-3D GRE, slice thickness $0.31 \mathrm{~mm}$ )

Table 2: Comparison of inter-marker distances (mm).

\begin{tabular}{ccccc}
\hline Markers & TS Image & \multicolumn{3}{c}{ MR Image } \\
& & $\mathbf{1}$ & $\mathbf{2}$ & $\mathbf{3}$ \\
\hline $1-2$ & 3.1 & $\mathbf{3 . 3}$ & 3.6 & 3.7 \\
$1-3$ & 5.7 & 5.0 & $\mathbf{5 . 5}$ & 6.1 \\
$1-4$ & 9.6 & 7.8 & $\mathbf{9 . 4}$ & 10.2 \\
$1-5$ & 8.9 & 8.3 & 8.6 & $\mathbf{8 . 9}$ \\
$1-6$ & 12.4 & 11.9 & $\mathbf{1 2 . 5}$ & 13.0 \\
$2-3$ & 4.9 & 4.6 & $\mathbf{4 . 9}$ & 5.3 \\
$2-4$ & 9.7 & 8.9 & $\mathbf{9 . 9}$ & 10.2 \\
$2-5$ & 6.9 & 6.4 & 6.4 & $\mathbf{6 . 5}$ \\
$2-6$ & 10.5 & 10.1 & $\mathbf{1 0 . 4}$ & 10.9 \\
$3-4$ & 5.0 & 4.6 & $\mathbf{5 . 2}$ & 5.3 \\
$3-5$ & 3.8 & 4.1 & $\mathbf{3 . 8}$ & 3.6 \\
$3-6$ & 6.9 & $\mathbf{7 . 1}$ & 7.2 & 7.2 \\
$4-5$ & 6.1 & 7.5 & $\mathbf{7 . 1}$ & 7.2 \\
$4-6$ & 6.9 & 8.8 & $\mathbf{7 . 9}$ & $\mathbf{7 . 9}$ \\
$5-6$ & 3.5 & $\mathbf{3 . 8}$ & 4.0 & 4.4 \\
\hline
\end{tabular}

Tab. 2 presents the inter-marker distances measured for three consecutive, and visually similar, MR images. Distances with the closest correspondence to the TS are highlighted. Based on these measurements, Fig. 1(d) corresponded best with the TS (Fig. 1(a)).

\section{Discussion}

This ex vivo investigation assessed the visibility of strandshaped markers in histopathology, MRI and macroscopic photographs. MR images were linked to histopathology slides, a cross-modality correlation which provided an indication as to the physical location of an MR slice within the sample.

Marker visibility in MRI was primarily influenced by factors unrelated to the markers themselves. For example, visibility was most impaired at the interface of the inserted muscle tissue due to small air inclusions at the skin-fat interface, and at various sites of tissue nonhomogeneity within the sample. The identification and tracking of markers through consecutive slices was facilitated by the strand-shaped nature and structured placement of markers, as well as by the isotropic sequence employed, which allowed high quality reformation of images in any plane.

The excellent contrast between markers and surrounding tissue enabled a precise measurement of marker positions. The digital TS photographs displaying markers (black) in paraffin embedded tissue (beige-pink) provided a valuable link in correlating histopathology and MRI observations. Inter-marker measurements in the x-direction (left to right) were more robust to the effects of sample distortion, as shrinkage along this axis was minimal.

Generally, the investigated fiducial marker in this study was clearly visible in histopathology, photography and MRI. Even though not all markers were visible in all sample slices, we were able to link sample slices to MR images, allowing a comparison of findings across modalities. In samples where fewer markers were visible, such a correspondence may carry increased uncertainty. The present study provides useful guidance for ongoing ex vivo research as well as future in vivo adaptations for forensic applications.

\section{Bibliography}

[1] Ward, A., Crukley, C. et. al.: Prostate: Registration of digital histopathologic images to in vivo MR images acquired using endorectal receive coil, Radiology, vol. 263, pp. 856-864, 2012.

[2] Yen, K., Vock, P. et. al.: Virtopsy: Forensic traumatology of the subcutaneous fatty tissue; Multislice Computed Tomography (MSCT) and Magnetic Resonance Imaging (MRI) as diagnostic tools, J Forensic Sci, vol. 49(4), pp. 799-806, July 2004.

[3] B. Dettmeyer, Forensic Histopathology: Fundamentals and Perspectives, Berlin-Heidelberg: Springer, 2011. 